\title{
Correction of prominent ears: techniques and complications
}

\author{
Jiyun Choi, MD, PhD \\ Department of Otorhinolaryngology, Chosun University College of Medicine, Gwangju, Rep. of Korea
}

Prominent ears are present in approximately $5 \%$ of the Caucasian population. It is characterized by autosomal dominant inheritance and commonly caused by 2 developmental defects: underdevelopment of the antihelical fold and overdevelopment of the conchal wall. A thorough preoperative evaluation includes examination of ear symmetry, size, shape, and projection. Mustarde first described a technique for creating an antihelical fold by utilizing permanent conchoscaphal mattress sutures. Furnas popularized a technique of conchal setback using permanent cochomastoidal suturing.

Keywords: prominent ears; mustard technique; furnas technique; complications

\section{Introduction}

Prominent ears are present in approximately $5 \%$ of the Caucasian population [1]. They are characterized by autosomal dominant inheritance and commonly caused by two developmental defects: underdevelopment of the antihelical fold and overdevelopment of the conchal wall [1]. The ear is a complex structure of cartilage and skin with many intricate involutions and folds. It includes five critical elements: the concha, helix, antihelix, tragus, and lobule. Parts of lesser importance include the antitragus, intertragic notch, and Darwin's tubercle [2]. The anatomical divisions of the ear are based in embryology, and the ear's origins are from the first (mandibular) and second (hyoid) branchial arches. These hillocks begin to fuse and form the external ear. Assigning a specific anatomic location on the ear to any given hillock is more speculative, but it is generally thought that the tragus helical crus and part of the helix develop from the mandibular arch, and the remainder of the external ear develops from the hillocks of the hyoid arch [2].

The ear attains approximately $85 \%$ of its adult size by 3 years of age [3]. Ear width matures in boys at 7 years and in girls at 6; ear length matures in boys at 13 years of age and in girls at 12 [4]. This implies that children 5-6 years of age may safely undergo otoplasty without compromising external ear growth.

The average height of an adult pinna is $5.5-6.5 \mathrm{~cm}$, with a width of 3.3-3.9 $\mathrm{cm}$. The helix is elevated $1-2 \mathrm{~cm}$ from the mastoid. The ideal auriculocephalic angle is 15-30 degrees; the conchocephalic and conchomastoid angles are 90 degrees [5]. The upper pole of the ear should fall at the level of the brow, whereas the inferior aspect of the lobe should fall at the level of the nasal ala. From the scalp, the helical rim of the ear commonly projects laterally $10-12 \mathrm{~mm}$ at the superior pole, $16-18$ $\mathrm{mm}$ at the midpoint, and 20-22 $\mathrm{mm}$ at the lobule [3].

The superficial temporal and postauricular branches of the external carotid system provide excellent blood supply to the pinna. The innervation to the external ear follows its embryologic branchial arch origins. It consists of the anterior and posterior branches of the great auricular nerve, which innervates the first branchial arch structures (tragus and helical crus), and the auriculotemporal nerve, which innervates the second 
branchial arch structures (helix, scapha, antihelix, concha, antitragus, external acoustic meatus, and lobule). The external auditory meatus also receives innervation from branches of the vagus and glossopharyngeal nerves (Fig. 1) [6].

\section{Preoperative evaluation}

A preoperative evaluation includes an examination of ear symmetry, size, shape, and projection. The physical exam of each auricle should be documented, with special attention to the status of the antihelix, and the depth and projection of the conchal bowl, as these two deformities most often coexist in a protruding ear. During preoperative evaluation, one should assess the following in a patient with prominent ears: (1) degree of antihelical folding; (2) depth of the conchal bowl; (3) plane of the lobule and deformity, if present; (4) angle between the helical rim and the mastoid plane; (5) quality and spring of the auricular cartilage [7]. The optimal timing of surgical correction depends on a rational approach based on auricular growth and the age of school matriculation. As the ear is nearly fully developed by the 6-7 years of age, correction may be performed at this time [8]. Existing deformities, postoperative course, realistic outcomes, and complications may be discussed with the patient and parents at that time.

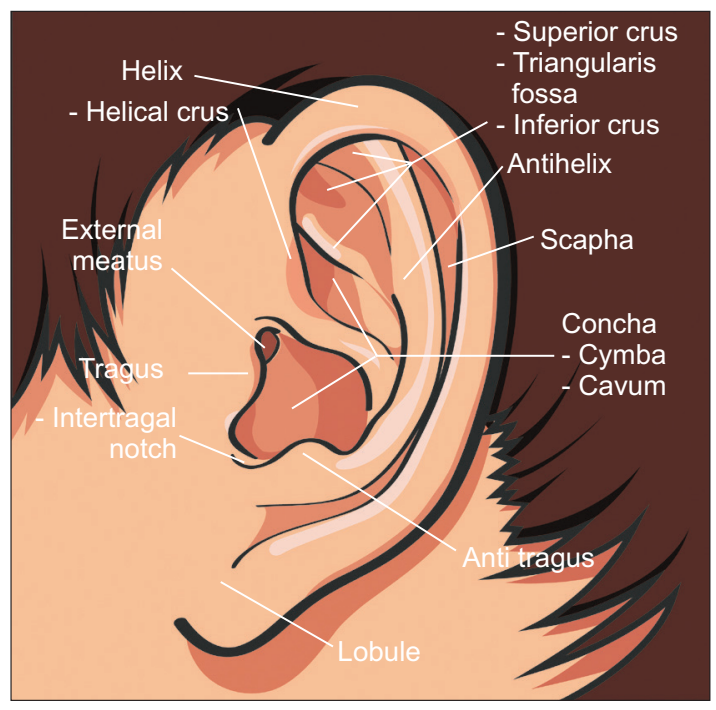

Fig. 1. Normal auricular anatomy.

\section{Otoplasty procedures}

\section{Mustard technique}

In 1963, Mustarde [9] first described the creation of an antihelical fold using permanent conchoscaphal mattress sutures. Several subtle refinements of this technique have been described since that time, but the fundamentals of the procedure remain unchanged.

Pediatric patients most commonly undergo this procedure under general anesthesia, and perioperative broad-spectrum antibiotics are administered. The face is prepped as a sterile field so that both ears can be visualized simultaneously. After infiltration with lidocaine $1 \%$ with epinephrine $1 / 100,000$, an elliptical or dumbbell-shaped skin excision is made that tapers at both ends for ease of closure. Typically, more skin is excised from the postauricular surface than from the mastoid to camouflage the resulting scar into the postauricular sulcus following setback.

Wide undermining is performed in the supraperichondrial plane almost up to the helical rim. The area of intended neoantihelix may be demarcated by placing 25 -gauge needles through the anterior auricular skin at the intended site of antihelix creation and bringing the needle out the posterior side. The cartilage is then marked with methylene blue. The Mustarde procedure consists of inserting three to four horizontal placement sutures (4-0 nylon) to permanently recreate the antihelix. The suture is placed through the cartilage and anterior perichondrium, but not the anterior skin. Outer cartilage bites of 1 $\mathrm{cm}$ are separated by $2 \mathrm{~mm}$. The distance between the outer and inner cartilage bites is $16 \mathrm{~mm}$.

The ear dressing is removed on the first postoperative day to check for hematomas, and then replaced for 3-4 additional days. Subsequently, a headband is worn continuously for 2 weeks and at night for an additional 4-6 weeks (Fig. 2) [9].

\section{Furnas technique}

In 1968, Furnas [10] popularized a conchal setback technique using permanent cochomastoidal suturing. This procedure is often used in conjunction with techniques to correct an absent antihelical fold, as described above.

The patient is prepped and draped in a manner similar to that described for antihelical fold correction. After infiltration of lidocaine $1 \%$ with epinephrine $1 / 100,000$, a fusiform incision is made in the postauricular region. The width of the incision is estimated by manually pushing the concha towards the mastoid. Care is taken to avoid excessive skin excision, as tension on 


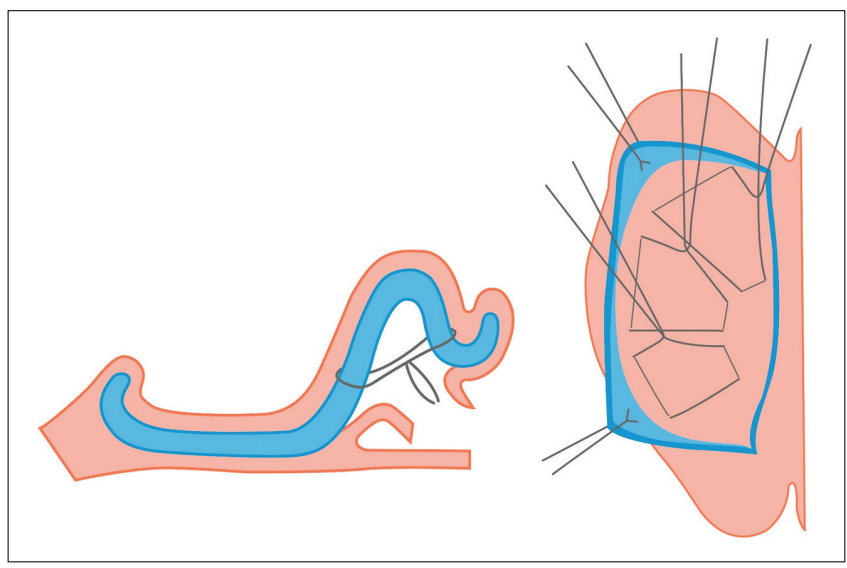

Fig. 2. Mustarde technique.

the wound predisposes to hypertrophic scar formation. Little to no skin excision is required inferior to the level of the antitragus. After skin excision, the soft tissue and postauricular muscle are excised from the postauricular sulcus. Sufficient soft tissue is excised to produce a pocket to receive the concha during suture placement.

The skin over the helix, antihelix, and concha is undermined with scissors, and permanent horizontal mattress sutures (e.g., 4-0 nylon) are placed at the lateral third of the concha cavum and concha cymba, parallel to the natural curve of the auricular cartilage. The sutures are placed through the cartilage and lateral perichondrium, but not the lateral auricular skin. At least three sutures are placed for adequate setback. The sutures are placed on what was the ascending wall of the concha. When tightened, they convert the wall into a longer floor of the concha. For long-term successful conchal reduction, suture bites of mastoid periosteum must be removed. Extremely thick cartilage frequently seen in older individuals may be weakened by excising small vertical ellipses. Importantly, conchomastoidal sutures must allow the concha to be set both medially and posteriorly, or external auditory canal stenosis can result.

The wound is irrigated and closed as after the Mustarde [10] technique. A mastoid dressing is placed, and subsequent postoperative management is the same as that of antihelical fold surgery (Fig. 3).

\section{Combined technique}

The postauricular pinna and postauricular sulcus are infiltrated with local anesthetic. The patient is then prepped and draped. The central portion of the desired antihelix is marked in three places using an 18-gauge needle dipped in methylene blue and used to tattoo the desired areas. The surgical proce-

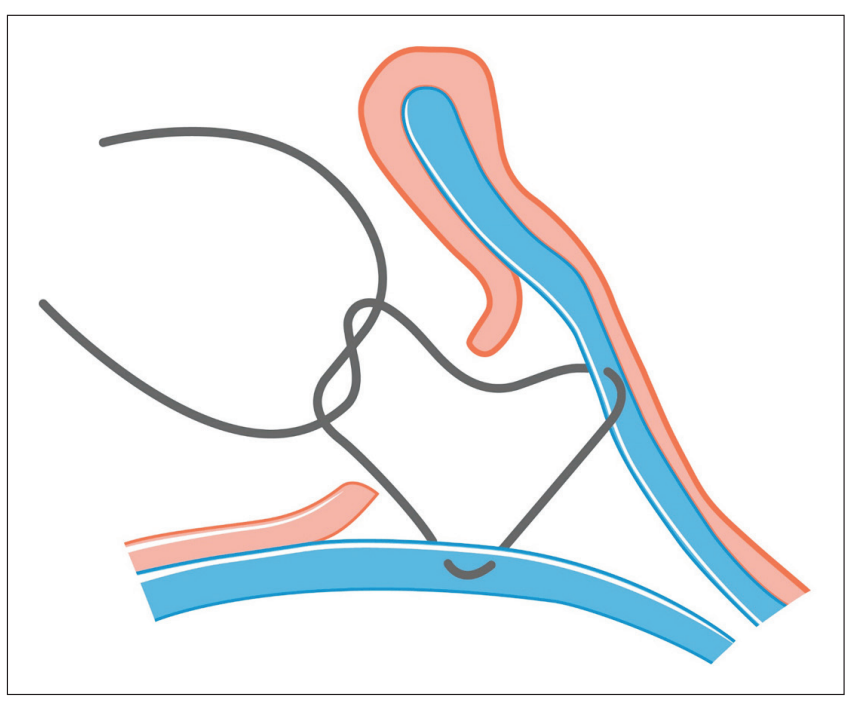

Fig. 3. Furnas technique.

dure is begun by removing a postauricular ellipse of skin. The posterior aspect of the auricular cartilage is exposed along the superior/inferior aspect of the pinna cartilage through helical rim. Small discs of cartilage are then scored and removed from the posterior aspect of the cavum conchae cartilage in areas where the patient has the most cartilage convexity. Conchalmastoid setback sutures are then placed. Using a 4-0 clear nylon suture, the conchal bowl is set back to its posterior medial extent. The antihelical fold is then created. Using the tattooed methylene blue markings, a horizontal mattress suture is placed with 4-0 nylon at the mid aspect of the desired antihelical fold. The perichondrium of the contralateral side is usually included in the surgical bite to avoid cheese-wiring of the suture through the cartilage over time. This is initially placed approximately 7 $\mathrm{mm}$ anterior to the blue marking and then $7 \mathrm{~mm}$ posteriorly in a symmetrical fashion. The skin is then closed with running locking 5-0 fast gut sutures. An identical procedure is performed on the contralateral side with careful attention to symmetry. After this procedure, the patient is placed in a secure dressing (Fig. 4).

\section{Complications}

Complications of otoplasty are not infrequently the consequences of poor surgical planning, although some may be unavoidable. Anterior surface irregularities, obliteration of the postauricular sulcus, and telephone ear deformity can be avoided with a meticulous surgical technique.

\section{Hematoma}

Hematomas occur in up to $3.5 \%$ of cases. Bleeding complications are typically an early complication due to incomplete 

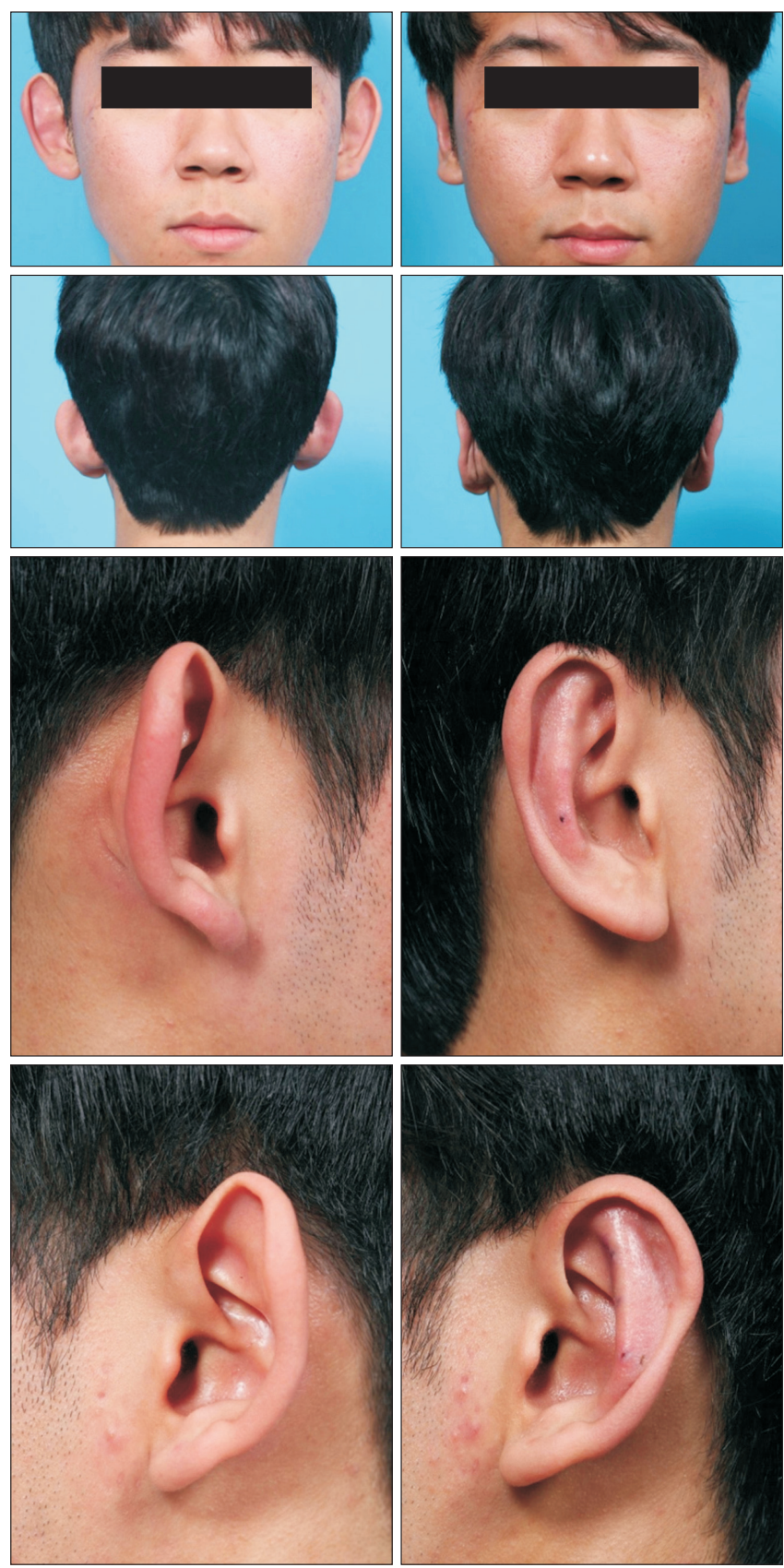

Fig. 4. A 23-year-old boy with protruding ears before (left) and after (right) otoplasty using the combined technique.

hemostasis during surgery. A common hallmark is bleeding around the dressing or severe unilateral pain. Urgent evacuation of the hematoma is critical to prevent fibrosis and, ultimately, permanent deformity of the auricle, known as "cauliflower ear." Careful hemostasis during hematoma evacuation should be obtained, and a drain and pressure dressing should be placed around the ear. The patient should be discharged on oral antibiotics and followed closely until the hematoma has completely resolved.

\section{Infection}

Wound infection occurs in $<5 \%$ of otoplasties. Sterile technique is critical for prevention, and most surgeons include prophylactic antibiotics. As with hematomas, prompt identification and treatment are essential to avoid permanent deformity. Infections may present as pain, erythema, swelling, and drainage. Any suspicion of infection should be treated aggressively with oral antibiotics. Management includes drainage and irrigation of the wound, followed by treatment with oral anti-pseudomonas antibiotics. Patients with severe infection may require IV antibiotics.

\section{Late complications}

Loss of correction or relapse of auricular deformity: This occurs more commonly after cartilage-sparing techniques. There are multiple technical causes of inadequate correction, including pulling of sutures over time, improper suture placement, failure to correct a deformity during surgery, failure to anchor sutures firmly on the mastoid periosteum, and failure to weaken noncompliant cartilage. Inadequate correction requires revision otoplasty.

Telephone and reverse telephone ear deformities: Telephone ear deformity occurs due to overcorrection in the middle third of the ear and relative under-correction of the superior and inferior poles. Reverse telephone ear deformity occurs when the middle third of the auricle remains prominent relative to the superior and inferior poles. Both deformities are avoidable with correct placement of the conchal setback sutures [11].

\section{Conclusion}

Otoplasty using the Mustard, Furnas, or combined techniques can result in successful outcomes, and these procedures are very useful for prominent ear patients. However, care must be taken to prevent complications such as hematoma, infection, under-correction, and telephone ear deformity.

\section{Acknowlegments}

This study was supported by research funds from Chosun University Hospital 2015. 


\section{Conflicts of interest}

The author has nothing to disclose.

\section{References}

1. Adamson PA, Strecker HD. Otoplasty techniques. Facial Plast Surg 1995;11:284-300.

2. Tolleth H. Artistic anatomy, dimensions, and proportions of the external ear. Clin Plast Surg 1978;5:337-45.

3. Adamson JE, Horton CE, Crawford HH. The growth pattern of the external ear. Plast Reconstr Surg 1965;36:466-70.

4. Farkas LG, Posnick JC, Hreczko TM. Anthropometric growth study of the ear. Cleft Palate Craniofac J 1992;29:324-9.
5. Rogers BO. Microtic, lop, cup and protruding ears: four directly inheritable deformities? Plast Reconstr Surg 1968;41:208-31.

6. Janis JE, Rohrich RJ, Gutowski KA. Otoplasty. Plast Reconstr Surg 2005;115:60e-72e.

7. Ellis DA, Keohane JD. A simplified approach to otoplasty. J Otolaryngol 1992;21:66-9.

8. Kotler HS, Robertson K, Tardy ME Jr. Pre- and postoperative management in otoplasty. Facial Plast Surg 1994;10:244-54.

9. Mustarde JC. The correction of prominent ears using simple mattress sutures. Br J Plast Surg 1963;16:170-8.

10. Furnas DW. Correction of prominent ears by conchamastoid sutures. Plast Reconstr Surg 1968;42:189-93.

11. Owsley TG, Biggerstaff TG. Otoplasty complications. Oral Maxillofac Surg Clin North Am 2009;21:105-18. 